\title{
Erratum to: Two New Clustering Algorithms for Vehicular Ad-Hoc Network Based on Ant Colony System
}

\author{
Mohammad Fathian' ${ }^{1}$ Gholam Reza Shiran ${ }^{2}$. \\ Ahmad Reza Jafarian-Moghaddam ${ }^{1}$
}

\section{Erratum to: Wireless Pers Commun (2015) 83:473-491 DOI 10.1007/s11277-015-2404-4}

The second author's affiliation was incorrect in the original publication. The correct affiliation is shown in this erratum.

The online version of the original article can be found under doi:10.1007/s11277-015-2404-4.

$\triangle$ Ahmad Reza Jafarian-Moghaddam

ar_jafarian@iust.ac.ir

1 School of Industrial Engineering, Iran University of Science and Technology, 16846-13114 Tehran, Iran

2 Faculty of Transportation and Traffic, Isfahan University, 81746-73441 Isfahan, Iran 